\title{
Ácido Acetilsalicílico e Clopidogrel na Prevenção da Trombose de Stent
} Acetylsalicylic Acid and Clopidogrel in the Prevention of Stent Thrombosis

\author{
Mariana Menegotto, Eduardo Maffini da Rosa, Marina Bertoni Guerra \\ Universidade de Caxias do Sul - UCS, Caxias do Sul, RS - Brasil
}

Nossa equipe de estudos em Cardiopatia Isquêmica parabeniza os autores pelo artigo "Efetividade e Segurança dos Stents Farmacológicos em um Serviço de Cardiologia em Curitiba - PR"1 e pelos resultados obtidos em seu serviço.

\section{Palavras-chave}

Stents farmacológicos, aspirina/administração \& dosagem, clopidogrel, trombose.
Sabe-se que a ocorrência de trombose de stent possui, muitas vezes, consequências catastróficas, como oclusão aguda do vaso, que chegam até mesmo a morte súbita ${ }^{2}$. A trombose de stent é um tema que nos interessa, em especial, na maneira de abordarmos tromboses tardias em stents farmacológicos. Por isso, gostaríamos de saber a opinião dos autores quanto ao uso rotineiro de ácido acetilsalicílico e clopidogrel, após o primeiro ano do implante de stent farmacológico, no intuito de evitar eventos decorrentes da trombose tardia de stent.

Correspondência: Mariana Menegotto •

Rua Travessao Solferino, 1156 - Cruzeiro - Caxias do Sul, RS - Brasil

E-mail: mmenegotto@gmail.com

Artigo recebido em 27/10/11; revisado recebido em 17/01/11; aceito em 17/01/11.

\section{Referências}

1. Braga CYY, Rivetti LA, Stella FP. Efetividade e segurança dos stents farmacológicos em um serviço de cardiologia em Curitiba-PR. Arq Bras Cardiol. 2010;95(1):3-11.
2. Campos CAHM, Ribeiro EE, Lemos PA, Falcão JL, Spadaro AG, Kajita LJ, et al. Perfil de segurança dos stents farmacológicos nas síndromes coronárias agudas: dados do Registro INCOR. Rev Bras Cardiol Invas. 2008;16(3):289-94. 